\title{
Esteróides Sexuais e Gonadotrofinas em Mulheres com e sem Carcinoma de Endométrio: Um Estudo Clínico Comparativo
}

\author{
Endogenous Sexual Steroids and Gonadotrophins in Women with or without Endometrial \\ Carcinoma: A Comparative Clinical Study
}

Valdir Tadini, Sylvia M.F. Brenna, Aarão Mendes Pinto-Neto, Lúcia Helena Simões da Costa Paiva,

Maurício de Sena Martins, Gustavo Antônio de Souza

\begin{abstract}
RESUM0
Objetivo: analisar os níveis dos esteróides sexuais endógenos e gonadotrofinas em mulheres com e sem câncer de endométrio.

Métodos: foi realizado estudo clínico-comparativo com 20 mulheres na pós-menopausa com câncer de endométrio e 20 mulheres na pós-menopausa, sem câncer de endométrio. A idade, o tempo de menopausa e o indice de massa corpórea foram utilizados como variáveis de emparelhamento. Os níveis plasmáticos dos esteróides sexuais endógenos foram medidos por métodos de radioimunoensaio e imunoenzimático. Para análise estatística utilizamos o teste "t" de Student.

Resultados: os niveis de androstenediona (A), testosterona total (t) e testosterona livre (TL) foram mais elevados nas mulheres com câncer de endométrio e os níveis de hormônio luteinizante $(\mathrm{LH})$ foram significativamente menores nessas mulheres. Também observamos que a razão (E1/A) mostrou valores significativamente menores no grupo de mulheres com câncer, ao passo que a razão (E2/E1) não apresentou diferenças nos dois grupos.

Conclusões: destacamos a potencialidade dos esteróides sexuais e gonadotrofinas na gênese do adenocarcinoma de endométrio em mulheres na pós-menopausa.
\end{abstract}

PALAVRAS-CHAVE: Menopausa. Endométrio. Câncer. Estrogênios. Androgênios. Dosagens hormonais.

Departamento de Tocoginecologia da Faculdade de Ciências Médicas da Universidade Estadual de Campinas e Hospital e Maternidade "Leonor Mendes de Barros" - São Paulo - SP

Correspondência:

Aarão Mendes Pinto Neto

UNICAMP - A/C Assessoria Técnica e Científica do CAISM

Rua Alexander Fleming, 101 - Cidade Universitária

"Zeferino Vaz"

13.081-970 - Campinas - SP

Tel. (019) 788-7325 e Fax (019) 287-5935

\section{Introdução}

A menopausa e o período subseqüente são marcados por mudanças significativas no padrão hormonal no organismo feminino. Essas alterações são bem nítidas quanto ao eixo hipotálamohipófise-ovário e também quanto à atividade da glândula supra-renal. 
Nos ovários observa-se a diminuição da produção de estrogênios acompanhada pela síntese de progesterona, decorrentes da diminuição dos compartimentos endócrinos e da sensibilidade dos folículos restantes aos estímulos gonadotróficos. Destaca-se, a partir dessa situação, a função ovariana androgênica com a produção de androstenediona e testosterona por sua porção estromal $^{8}$.

Em razão do hipoestrogenismo as atividades hipofisária e hipotalâmica se mantêm exacerbadas, com persistência de altos níveis de FSH, LH e GnRH. Por fim, a supra-renal passa a assumir 95\% da produção de androstenediona e 100\% de sulfato de deidroepiandrostenediona (SHDEA) ${ }^{8}$.

Essas mudanças conferem à mulher na pósmenopausa um perfil estrogênico e androgênico característicos. Dentre os estrogênios, além de suas quedas globais, observa-se uma inversão entre os niveis séricos das frações estrogênicas, resultando na predominância da estrona em relação ao estradiol, diferente do encontrado na mulher jovem ${ }^{4}$.

Estima-se que cerca de 10\% das mulheres pós-menopausadas não apresenta o padrão habitual de evolução dos esteróides sexuais como mencionado, mas, ao contrário, apresenta a elevação dos níveis de estrogênios endógenos tornando-o o principal grupo de risco para o câncer de endométrio ${ }^{8}$.

Além disso, observamos nesse grupo de mulheres queda de produção da progesterona, importante fator de proteção contra o câncer de endométrio. Dessa maneira, aceita-se na atualidade que os estrogênios endógenos exercem um papel importante na fisiopatologia do câncer de endométrio em mulheres pós-menopausadas ${ }^{13}$. Vários estudos já relataram a associação entre a elevação dos esteróides endógenos e o câncer de endométrio ${ }^{9}$ e, atualmente, essa associação volta a ocupar lugar importante no estudo da etiologia dessa patologia ${ }^{10,13}$.

Dessa maneira, o objetivo principal deste estudo foi avaliar os níveis plasmáticos de esteróides sexuais endógenos e das gonadotrofinas em mulheres pós-menopausadas com e sem adenocarcinoma do endométrio.

\section{Pacientes e Métodos}

Foram sujeitos do estudo 40 mulheres assistidas no Ambulatório de Ginecologia Especializada do Hospital Maternidade "Leonor Mendes de Barros", no período de dezembro de 1995 a dezembro de 1997, divididos em dois grupos: o grupo A foi composto de 20 mulheres com diagnóstico de adenocarcinoma de endométrio obtido por curetagem uterina, e o grupo B foi composto por 20 mulheres sem adenocarcinoma de endométrio pareadas por idade, tempo de menopausa e índice de massa corpórea.

Os critérios de inclusão considerados foram: ter mais de 45 anos de idade; estar menopausada, ou seja, mais de 12 meses consecutivos sem menstruação; apresentar diagnóstico anatomopatológico de adenocarcinoma endometrióide do corpo uterino obtido por material de curetagem uterina fracionada.

Foram excluídas do estudo mulheres em uso de terapia de reposição hormonal atual ou prévia, as submetidas à ooforectomia uni ou bilateral ou a tratamento oncológico prévio.

A variável dependente considerada foi o diagnóstico histopatológico de adenocarcinoma endometrióide do corpo uterino, obtido por curetagem uterina fracionada. Foram incluídas no Grupo B (controle) as mulheres que apresentaram espessura endometrial menor ou igual a $5 \mathrm{~mm}$ à ultra-sonografia transvaginal ${ }^{11}$, sem sangramento na pós-menopausa ou outros sinais sugestivos de neoplasia genital.

As variáveis independentes foram os niveis de estrogênio, os niveis de androgênios, os niveis de gonadotrofinas, a razão estrona/ androstenediona (E1/A); e a razão estradiol/ estrona (E2/E1).

As variáveis controles foram a paridade, a idade na menopausa e a idade na menarca. As pacientes nos dois grupos foram pareadas por idade, tempo de menopausa e índice de massa corpórea ${ }^{7}$.

Coletou-se $30 \mathrm{ml}$ de sangue retirado de veia antecubital, que foi depositado em frascos heparinizados e esterilizados. Posteriormente, foi centrifugado e o plasma obtido ficou armazenado a 20 graus centígrados negativos até o processamento simultâneo de todas as amostras. As amostras foram analisadas pelos método de radioimunoensaio (da Diagnostic Products Corporation): E1, T, TL, SDHEA e A e imunoenzimático (AIA-PACK da TOSOH II): E2, FSH e LH. Foram efetuadas dosagens dos seguintes hormônios, em cada paciente: estrona, estradiol, testosterona total e livre, androstenediona, sulfato de deidroepiandrostenediona, hormônio folículoestimulante e hormônio luteinizante.

Para análise estatística foi utilizado o teste de comparação das médias, teste " $t$ " de Student com nível de significância de $5 \%{ }^{16}$.

\section{Resultados}

Os dois grupos foram bastante homogêneos quanto à idade, índice de massa corpórea, tempo 
de menopausa (variáveis de pareamento), porém, apresentaram pequenas diferenças em relação à idade na menopausa e idade na menarca e importante variação em relação à paridade (Tabela 1).

Tabela 1 - Características clínicas das mulheres com e sem câncer de endométrio. (medianas e valores mínimos e máximos)

\begin{tabular}{lcc} 
Características & $\begin{array}{c}\text { Sem câncer } \\
\text { mediana (min-max) }\end{array}$ & $\begin{array}{c}\text { Com câncer } \\
\text { mediana (min-max) }\end{array}$ \\
\hline Idade (anos) & $65,5(55-76)$ & $65,5(53-77)$ \\
Índice de massa corpórea $\left(\mathrm{kg} / \mathrm{m}^{2}\right)$ & $27,8(21,09-40,16)$ & $26,8(19,3-38,8)$ \\
Tempo de menopausa (anos) & $18(1-34)$ & $17(2-34)$ \\
Idade na menopausa (anos) & $50(39-65)$ & $50(40-62)$ \\
Idade na menarca (anos) & $14(10-18)$ & $13(10-16)$ \\
Paridade & $5(0-14)$ & $2,5(0-10)$ \\
\hline
\end{tabular}

A média dos níveis plasmáticos de E1 e E2 em mulheres com e sem câncer de endométrio não apresentou diferenças significativas. Observamos ainda que as médias de E1 e E2 são menores nas mulheres com câncer (Tabela 2).

As médias dos níveis plasmáticos de $\mathrm{A}, \mathrm{T}$ e TL em mulheres com câncer de endométrio foram maiores do que as das mulheres sem câncer de endométrio, aproximando-se muito da significância, Os níveis de A apresentaram a maior diferença. Por outro lado, dentre os androgênios não encontramos diferenças significativas entre as médias dos níveis de SDHEA em mulheres com e sem câncer de endométrio (Tabela 3 ).

Tabela 2 - Níveis plasmáticos de estrogênios em mulheres com e sem câncer de endométrio (médias e desvios padrão)

\begin{tabular}{|c|c|c|c|c|c|c|}
\hline \multirow{2}{*}{$\begin{array}{l}\text { Estrogênios } \\
\text { (pg/ml) }\end{array}$} & Sem câncer & \multicolumn{2}{|c|}{ Com câncer } & \multirow[b]{2}{*}{$\Delta$} & \multirow[b]{2}{*}{ 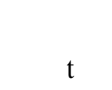 } & \multirow[b]{2}{*}{$\mathrm{p}$} \\
\hline & média DP & média & DP & & & \\
\hline E1 & $21,11 \quad 16,25$ & 17,46 & 16,29 & $-3,65$ & $-0,68827$ & 0,24979 \\
\hline F2 & $42,12 \quad 29,23$ & 39,12 & 26,13 & $-3,00$ & $-0,33231$ & 0,37164 \\
\hline
\end{tabular}

Tabela 3 - Níveis plasmáticos dos androgênios em mulheres com e sem câncer de endométrio (médias e desvios padrão)

\begin{tabular}{|c|c|c|c|c|c|c|c|}
\hline \multirow{2}{*}{$\begin{array}{l}\text { Androgênios } \\
(\mathrm{pg} / \mathrm{ml})\end{array}$} & \multicolumn{2}{|c|}{ Sem câncer } & \multicolumn{2}{|c|}{ Com câncer } & \multirow[b]{2}{*}{$\Delta$} & \multirow[b]{2}{*}{$\mathrm{t}$} & \multirow[b]{2}{*}{$p$} \\
\hline & média & DP & média & DP & & & \\
\hline (ng/ml) & 0,90 & 0,39 & 1,50 & 1,53 & $+0,60$ & 1,66835 & 0,05582 \\
\hline
\end{tabular}

SDHEA(ng/ml) $\quad 612,31578,99 \quad 681,71 \quad 485,26 \quad+69,40 \quad 0,48614 \quad 0,31621$

\begin{tabular}{llllllll}
$\mathrm{T}(\mathrm{ng} / \mathrm{dl})$ & 36,56 & 12,40 & 45,41 & 23,21 & $+8,85$ & 1,62105 & 0,06074 \\
$\mathrm{TL}(\mathrm{pg} / \mathrm{ml})$ & 1,23 & 0,66 & 1,75 & 1,51 & $+0,52$ & 1,61740 & 0,06113 \\
\hline
\end{tabular}

As médias dos niveis plasmáticos das gonadotrofinas (FSH e $\mathrm{LH}$ ) foram menores em mulheres com câncer de endométrio, com significância estatística em relação aos valores de FSH e LH (Tabela 4).

As mulheres com câncer apresentaram menores médias dos valores da razão E1/A, com diferença significante estatisticamente. Os dois grupos apresentaram as médias dos valores de E2/ E1 bem próximas, apesar do desvio padrão em mulheres sem câncer ser praticamente o dobro do outro e maior que as médias (Tabela 5).

Tabela 4 - Níveis plasmáticos das gonadotrofinas em mulheres com e sem câncer de endométrio (médias e desvios padrão)

\begin{tabular}{|c|c|c|c|c|c|c|c|}
\hline \multirow{2}{*}{$\begin{array}{l}\text { Gonadotrofinas } \\
(\mathrm{mUI} / \mathrm{ml})\end{array}$} & \multicolumn{2}{|c|}{ Sem câncer } & \multicolumn{2}{|c|}{ Com câncer } & \multirow[b]{2}{*}{$\Delta$} & \multirow[b]{2}{*}{$\mathrm{t}$} & \multirow[b]{2}{*}{$\mathrm{p}$} \\
\hline & média & $\mathrm{DP}$ & média & DP & & & \\
\hline FSH & 69,35 & 34,40 & 52,88 & 25,85 & $-16,47$ & $-1,85698$ & 0,03944 \\
\hline $\mathrm{LH}$ & 23,28 & 12,80 & 15,45 & 10,80 & $-7,83$ & $-2,56228$ & 0,00952 \\
\hline
\end{tabular}

Tabela 5 - Razões entre as médias dos valores de E1/A e E2/E1 das mulheres com e sem câncer de endométrio

\begin{tabular}{|c|c|c|c|c|c|c|c|}
\hline \multirow[t]{2}{*}{ Razão } & \multicolumn{2}{|c|}{ Sem câncer } & \multicolumn{2}{|c|}{ Com câncer } & \multirow[b]{2}{*}{$\Delta$} & \multirow[b]{2}{*}{$\mathrm{t}$} & \multirow[b]{2}{*}{$P$} \\
\hline & média & DP & média & $\mathrm{DP}$ & & & \\
\hline $\mathrm{E} 1 / \mathrm{A}$ & 0,026 & 0,025 & 0,014 & 0,012 & $-0,012$ & $-1,74295$ & 0,04875 \\
\hline E2/E1 & 4,003 & 6,068 & 4,124 & 3,381 & $+0,121$ & $+0,07145$ & 0,47189 \\
\hline
\end{tabular}

\section{Discussão}

O objetivo principal desta investigação foi avaliar os niveis de alguns esteróides sexuais endógenos e das gonadotrofinas em mulheres com e sem adenocarcinoma de endométrio. Analisando os dados obtidos, observamos que os níveis plasmáticos de androstenediona, testosterona total e testosterona livre foram mais elevados em mulheres com câncer de endométrio ao passo que os de gonadotrofinas, bem como da razão E1/A, foram mais baixos nesse grupo.

Cabe destacar que as pacientes incluídas neste estudo foram pareadas uma a uma, por idade, tempo de menopausa e índice de massa corpórea, variáveis que, sabidamente, em maior ou menor grau, poderiam influir nos resultados. Notamos, também, semelhança em relação à idade na menarca e à idade na menopausa, porém, os dois grupos apresentaram-se bem diferentes em relação à paridade, pois para cada mulher nulípara sem 
câncer tivemos cinco mulheres nulíparas com câncer.

Em relação aos estrogênios endógenos analisados (E1 e E2), os resultados obtidos são discordantes com os da literatura que, em geral, relata elevações dos níveis plasmáticos de estrona em mulheres na pós-menopausa, principalmente, naquelas com câncer de endométrio ${ }^{10,13}$.

Observamos, em relação aos níveis de esteróides sexuais que, se por um lado os níveis dos estrogênios endógenos (estrona e estradiol) não apresentaram diferenças significativas nos dois grupos por outro, os niveis de androgênios, principalmente a androstenediona (A), a testosterona total (T) e a testosterona livre (TL) apresentaram-se maiores nas mulheres com câncer de endométrio. Não encontramos diferenças significativas entre os grupos, em relação aos níveis de SDHEA, o que é semelhante aos resultados contidos na literatura ${ }^{6,14,13}$.

Em relação às gonadotrofinas, encontramos níveis plasmáticos significativamente menores de LH e FSH entre as mulheres com câncer, o que pode ser considerado um resultado inesperado. Sabe-se que níveis plasmáticos elevados de $\mathrm{LH}$, geralmente, acompanham-se de maior produção de androgênios pelo estroma ovariano na pós-menopausa. Entretanto, essa relação não pôde ser confirmada neste estudo e nem no estudo de Brown $^{2}$. Esse autor sugeriu que a insuficiente produção de $\mathrm{LH}$ em mulheres com câncer de endométrio pode ser em virtude de antecedentes pessoais de distúrbios ovulatórios com decréscimo da fertilidade e à exposição prolongada da hipófise aos níveis constantemente elevados dos estrogênios.

Em relação aos níveis plasmáticos de $\mathrm{FSH}$ os nossos resultados são concordantes com as observações de Benjamin e Deutsch ${ }^{1}$, que também observaram menores niveis plasmáticos de FSH em mulheres com câncer de endométrio.

Nesta casuística vimos também que a razão estrona/androstenediona (E1/A) apresentou-se menor nas mulheres com câncer, o que discorda dos resultados obtidos por Nyholm et al. ${ }^{10}$, que mostram uma elevação desses valores, principalmente devido a um aumento dos niveis de estrona.

A razão estradiol/estrona (E2/E1) mostrouse bem próxima nos dois grupos. Porém, metade das mulheres com câncer tinha a razão E2/E1 maior ou igual a 3,00, o que difere do esperado que seria a inversão do padrão estrogênico da pós-menopausa, com predominância da estrona sobre o estradiol numa relação de $2: 1$ a $3: 1^{14}$.

É clássica também a associação entre a tríade: obesidade, diabete e hipertensão arterial com o câncer de endométrio; porém, encontramos muita controvérsia na literatura em relação a essas situações clínicas supostamente predisponentes. O que há de definitivo é que, de alguma forma, ocorreria nessas mulheres uma aceleração da conversão periférica dos esteróides sexuais e/ou uma produção aumentada de androgênios pela glândula supra-renal e, ainda, uma maior resposta dos receptores citoplasmáticos de estrogênios no endométrio ${ }^{5}$.

Outros estudos sugerem que não apenas os níveis plasmáticos de estrogênios, mas sim a sua relação com a androstenediona (E1/A), refletiria uma alteração do ritmo de aromatização dos androgênios para a sua conversão em estrogênios. A relação entre eles, principalmente a razão $\mathrm{E} 1 / \mathrm{E} 2$ ou E2/E1, que mostra a proporção entre o estrogênio mais importante da mulher na menacme (estradiol) e o estrogênio da mulher na pósmenopausa (estrona), seria mais importante na explicação da gênese do câncer de endométrio ${ }^{10,14}$.

Alguns autores, em concordância com o nosso estudo, denotam uma maior importância da combinação entre os estrogênios e androgênios endógenos e das gonadotrofinas, ou seja, uma alteração global desses, predominando as variações mais significantes nos níveis dos androgênios ${ }^{6,9,12,15}$.

Devemos ressaltar que estudos de comparação dos níveis de esteróides sexuais endógenos e gonadotrofinas em pacientes com e sem câncer de endométrio apresentaram resultados diferentes de acordo com a metodologia empregada.

Nyholm e cols. $(1993)^{10}$, utilizando um desenho de estudo igual ao por nós empregado, com cerca de 60 mulheres em cada grupo, observaram diferenças significativas em relação aos níveis de estrona, estradiol, estradiol livre, androstenediona e razão E1/A, que se apresentaram aumentados no grupo de mulheres com câncer. Com exceção da elevação dos níveis da androstenediona, não encontramos essas alterações em nosso estudo.

Recentemente, Potischman et al. ${ }^{13}$, em estudo prospectivo com cerca de 200 mulheres em cada grupo com e sem câncer de endométrio, encontraram um aumento dos níveis de estrona e androstenediona. Esse resultado reforça o sugerido por Bulun et al. ${ }^{3}$ de que um acréscimo da produção de estrona, por aumento do pool circulante de androstenediona ou rotas alternativas de produção de estrona, é importante fator associado ao aparecimento da neoplasia endometrial.

Apesar dos resultados não serem definitivos, associados aos dados da literatura, não cabe dúvida de que as variações dos níveis dos esteróides sexuais endógenos e gonadotrofinas na pós-menopausa, em pacientes sem terapia de 
reposição hormonal, poderá levar à suspeita da presença de câncer do endométrio e, portanto, essas mulheres devem ser consideradas como de alto risco e merecem controle rigoroso durante suas vidas.

\section{SUMMARY}

Objective: To analyze the levels of endogenous sexual steroids and gonadotrophin in women with and without endometrial cancer.

Methodology: We developed a clinical comparative study on 20 postmenopausal women with endometrial cancer and 20 postmenopausal women without endometrial cancer. The age, the postmenopausal time and the index of body mass were used as matching variables. The plasma levels of the endogenous sexual steroids were measured using radioimmunoassay and immunoenzymatic methods. For the statistic analysis we used the Student's t test.

Results: The levels of androstenedione (A), total testosterone $(t)$ and free testosterone (TL) were higher in the women with endometrial cancer, and those of the luteinic hormone $(\mathrm{LH})$ were significantly lower values in these women. We also observed that the ratio (E1/A) showed significantly lower in the group of women with cancer, while the ratio (E2/E1) did not present any differences between groups.

Conclusions: We emphasize the potentiality of sexual steroids and gonadotrophins in the genesis of endometrial adenocarcinoma in postmenopausal women.

KEY WORDS: Menopause. Endometrium. Cancer. Estrogen. Androgen. Hormone dosage.

\section{Referências}

1.Benjamin F, Deutsch S. Plasma levels of fractionated estrogens and pituitary hormones in endometrial carcinoma. Am J Obstet Gynecol 1976; 126: 638-47.

2.Brown R. Clinical features associated with endometrial carcinoma. Obstet Gynaec Brit Comnwlth 1974; 81: 933-41.

3.Bulun SE, Economos K, Miller D, Simpson ER. CYP 19 (aromatase cytochrome P450) gene expression in human malignant endometrial tumors. J Clin Endocrinol Metab 1994, 79:1831-4.
4.Gambrell Jr RD. Pathophysiology and epidemiology of endometrial cancer. In: Lobo RA, editor. Treatment of the Postmenopausal Woman: Basic and Clinical Aspects. New York: Raven, 1994. p.355-62.

5.Gusberg SB. The individual at high risk for endometrial carcinoma. Am J Obstet Gynecol 1976; 126:535-42.

6.Judd HL, Davidson BJ, Frumar AM, Shamonki IM, Lagasse, LD, Ballon SC. Serum androgens and estrogens in postmenopausal women with and without endometrial cancer. Am J Obstet Gynecol 1980; 136:859-66.

7.Keys A, Fidanza F, Karvonen MJ, Kimura N, Taylor HL. Indices of relative weight and obesity. J Chron Dis 1972; 25:329-43.

8.Longcope C. The endocrinology of the menopause. In: Lobo RA, editor. Treatment of the Postmenopausal Woman: Basic and Clinical Aspects. New York: Raven; 1994. p.47-53.

9.Nagamani M, Hannigan EV, Dillard Jr EA, Vandinh T. Ovarian steroid secretion in postmenopausal women with and without endometrial cancer. $\mathbf{J}$ Clin. Endocrinol. Metab. 1986; 62:508-12.

10.Nyholm HCJ, Nielsen AL, Lyndrup J, Dreisler A, Hagen C, Haug E. Plasma oestrogens in postmenopausal women with endometrial cancer. $\mathbf{B r}$ J Obstet Gynaecol 1993; 100:1115-9.

11.Parsons AK. Sonography: a new method to evaluate the endometrium. Menopausal Medicine 1993;1:9-12.

12.Pettersson B, Bergstron R, Johansson EDB. Serum estrogens and androgens in women with endometrial cancer. Gynecol Oncol 1986; 25:223-33.

13.Potischman N, Hoover RN, Brinton LA, Siiteri P, Dorgan JF, Swanson CA et al. Case-Control study of endogenous steroid hormones and endometrial cancer. J Natl Cancer Inst 1996; 88:1127-35.

14. Rodrigues de Lima G, Baracat EC, Girão MJC. Aspectos endócrinos do carcinoma do endométrio. In: Rodrigues de Lima G, Baracat EC, Girão MJC, editores. Ginecologia Endócrina. São Paulo: Atheneu; 1995. p.315-9.

15.Siiteri PK, Schwartz BE, Mac Donald PC. Estrogen receptors and the estrone hypothesis in relation to endometrial and breast cancer. Gynecol Oncol 1974; 2:228-38.

16.Soares JF, Bartmann FC. Introdução aos métodos estatísticos em oncologia. Belo Horizonte: Sociedade Brasileira de Oncologia Clínica; 1985, 99p. 